\title{
ACTITUDES LINGUISTICAS HACIA DOS VARIEDADES DE HABLA: VALLE CENTRAL Y GUANACASTE
}

\author{
Marjorie Arrieta Molina \\ Carla Victoria Jara Murillo \\ Covadonga Pendones de Pedro
}

\begin{abstract}
A description of the existing variation in the linguistic attitudes among high school students towards two speech varieties that can be clearly pointed out in Costa Rica: Central Valley speech and Guanacaste speech. The theoretical and methodological guidelines set forth by William Labov (1972) are followed.
\end{abstract}

\section{INTRODUCCION}

El campo de las actitudes lingüísticas ha sido poco desarrollado en el marco de las investigaciones sobre las variedades de habla regionales en Costa Rica. Esta carencia ha motivado recientes trabajos bajo las directrices de la sociolingüística, disciplina que ha venido tomando un lugar predominante en los estudios lingüísticos y que postula que las variaciones en el habla, determinadas socialmente, pueden ser sistematizadas y cuantificadas.

La presente investigación, siguiendo los lineamientos de este creciente impulso, pretende analizar las actitudes que surgen en los hablantes de dos comunidades lingü ísticas en relación con ambas variedades, específicamente la del Valle Central y la de la región de Guanacaste. Para tal efecto, entendemos por comunidad lingü ística, siguiendo a Labov (1972a: 120), aquella que:

“...no viene definida tanto por un acuerdo señalado sobre el uso de elementos lingü ísticos como por la participación en un conjunto de normas compartidas; tales normas pueden ser observadas en tipos manifiestos de comportamiento evaluativo, y por la uniformidad de modelos abstractos de variación que son invariables respecto de particulares niveles de uso".

A lo largo de este trabajo, llamaremos "variedad A" a la del Valle Central y "variedad B" a la de Guanacaste.
2. VARIEDADES REGIONALES: VALLE CENTRAL (A) Y GUANACASTE (B)

\subsection{Reseña histórica}

A finales del siglo XVIII, se habían fundado ya las que serían las cuatro principales ciudades de Costa Rica: Cartago, que se traslada en 1575 al Valle del Guarco; y Villa de la Boca del Monte (luego San José, fundada en 1739), Villa Vieja (luego Heredia, fundada en 1763) y Villa Hermosa (luego Alajuela, fundada en 1784) en el Valle Central, antiguamente llamado Valle de Occidente.

En cuanto a Guanacaste, Rivas se había fundado a principios del siglo XVII; entre ella y el Valle de Occidente solamente había una población: $\mathrm{Ni}$ coya, Liberia, actualmente capital de Guanacaste, nació de la necesidad de los finqueros de la región de crear un punto que uniera Rivas, Nicoya y el actual Valle Central. Se toma el año de 1769 como fecha de la fundación de Liberia. La región de Guanacaste estuvo poblada por señores feudales nicaragüenses, por lo que siempre sostuvo una estrecha relación con ese país.

En 1812, y en virtud de que el número de habitantes de Costa Rica era insuficiente para que se pudiera elegir diputado en las Cortes, las autoridades del Reino dispusieron que se tomaran en cuenta los habitantes del Partido de Nicoya; y ya para 1822 se tomaban como parte de Costa Rica los partidos de Guanacaste y de Nicoya. 


\subsection{Diferencias entre las variedades}

Fundamentalmente, las diferencias se presentan en los niveles léxico y fonético. A continuación esbozamos las diferencias fonéticas más sobresalientes de ambas variedades(1)..

\section{Variedad A}

- Relajamiento y ensordecimiento de la vocal final de palabra.

- Realización del grupo [tr] como una secuencia africada ápicoprepalatal.

- Realización de [ $\mathrm{J}$ ] como una vibrante fricativa sonora y de [ ] ] como una friacativa sorda.

\section{Variedad B}

- Mayor frecuencia de aspiración fortis de /s/.

- Realización del grupo [ tr] como una secuencia oclusiva vibrante.

- Realización de la [ $\overline{\mathrm{r}}$ ] como una vibrante múltiple sonora.

- Lenición de la palatal fricativa/ $/$ /intervocálica: 'muchachillo > muchachío'.

- A causa de esta lenición, se da un fenómeno de ultracorrección en palabras como'tía > tiya', donde se introduce una yod.

\section{Marco teórico}

\subsection{Las actitudes lingüisticas}

La desigualdad lingüística es un hecho comprobado por los estudios sociolingü ísticos. Hudson (1981: 105-7) señala tres tipos de desigualdad lingü ística:

1. Desigualdad subjetiva: consiste en lo que la gente piensa del habla de los demás y corresponde al área de los prejuicios lingüísticos, en la cual se sitúa la presente investigación.

2. Desigualdad estrictamente lingü ística: referida a los elementos lingü ísticos que una persona conoce.

3. Desigualdad comunicativa: trata de cómo utilizar los elementos lingü ísticos para comunicarse eficazmente.

Suelen asociarse valores a características no lingü ísticas de habla, de manera que una variedad que posea una característica altamente valorada se contrapone a otra habla que tenga la característica considerada de bajo valor.
Las características valoradas varían de una comunidad lingü ística a otra. Estos valores asociados al habla tienen una conexión directa con los símbolos de pertenencia a un grupo, de tal forma que los individuos utilizan su habla con el fin de identificarse con el grupo social al que pertenecen o al que quisieran pertenecer.

El fenómeno contrario es la inseguridad lingüística (Labov, 1972a: 173), que se da cuando un grupo determinado cree que habla peor que otros, asociando valores negativos a su propia habla. En la educación formal se adopta la variedad estándar, que es la determinada por el grupo de mayor prestigio.

Según estos planteamientos, las pruebas de reacción subjetiva como la que utilizamos en esta investigación, por lo general muestran claramente las diferencias de actitud que los miembros típicos de un grupo dado tienen en relación con las diferencias de pronunciación. Luego de escuchar la voz y con ella de pronunciación del hablante, el juez dará una descripción de ese hablante, pero basado únicamente en sus concepciones estereotipadas.

\subsection{Aportes de Labov}

En Métodos sociolingüisticos (1972), Labov ha reunido diversas investigaciones con las cuales ha logrado resultados sorprendentes. En el campo de las actitudes lingüísticas, este autor ha establecido índices de comportamiento general para las comunidades lingü ísticas estudiadas planteándolos en la etapa de evaluación del campo lingüístico, en la cual se ponen en evidencia las actitudes generales y las aspiraciones de los informantes. Así, las actitudes lingü ísticas de una comunidad favorecen o desfavorecen las condiciones para que un cambio se propague o se limite.

Las fuerzas sociales que intervienen en el cambio tienen que ver con las presiones "desde arriba" o "desde abajo". Estas hacen referencia a las presiones por debajo del nivel de la capacidad conciente del hablante. La clase media es decisiva en estas presiones puesto que los hablantes de clase media baja superan al grupo de mayor estatus en su tendencia a usar formas consideradas como correctas. Esta pauta de superación aparece como desviación del comportamiento que muestran otras clases.

La clase media baja se ve afectada por su gran inseguridad lingüística, al tratarse de una clase en "ascenso" social. Esto se demuestra por la gran fluctuación de la variación estílistica, su hipersen- 
sibilidad ante las formas relegadas que ellos mismos usan, y la percepción errónea de su propio discurso.

La edad se establece como una variante significativa puesto que de los cuatro a los trece años, los niños siguen las pautas del grupo de compañeros. Parece que en este período es cuando las pautas automáticas de producción lingü ística quedan establecidas en su conjunto: cualquier regla, cualquier costumbre adquirida se mantiene por un control auditivo adicional.

En el primer año de secundaria el adolescente adquiere el conjunto de normas evaluativas y se hace sensible a la significación de su propia habla. Aun así, los adolescentes de las clases bajas, a la hora en que adquieren un buen conocimiento de las normas, ya no son capaces de modificar su habla original. Esto lo demuestra el hecho de que la clase media baja varía significativamente del habla espontánea al habla formal.

\section{Metodología y proceso de la investigación}

\subsection{Confección de instrumento}

En la etapa previa a la confección del instrumento que serviría a los jueces para evaluar a los hablantes, se recogió una cantidad de adjetivos evaluativos sobre las dos variedades. Siguiendo el método directo, se les hizo a hablantes de una y otra variedad las siguientes preguntas:

1. ¿Qué le parece la forma en que habla la gente de Guanacaste?

2. ¿Qué le parece la forma en que habla la gente del Valle Central?

De esta manera, se estableció un corpus de adjetivos con sus antónimos que fueron dispuestos en escalas de siete espacios, siguiendo el modelo de la "escala de diferencial semántico" de Osgood (1957). Se consideró el adjetivo positivo como polo 1 y el negativo como polo 7.

Resultaron las siguientes 12 escalas, correspondientes a tres ejes:

\section{I eje: estatus social}

1- urbano-campesino

2- adinerado-pobre

3- elegante-polo

4- instruido-ignorante

II eje: capacidad lingüística
5- habla bajito-habla fuette

6- habla seguido-habla cortaido

7- habla lento-habla rápido

8- no habla cantado-habla cantado

III eje: emotivo

9- agradable-desagradable

10- amable-grosero

11- habla como yo-habla diferente

12- habla correctamente-habla incorrectamente

En el cómputo de los datos (2), se desechó el eje de capacidad lingü ística por no proveer datos significativos; los rubros restantes fueron reagrupados de la siguiente forma:

1- Escala urbano-campesino

2- Eje de estatus (escalas: pobre-adinerado, elegante-polo e instruido-ignorante)

3- Eje emotivo (escalas: agradable-desagradable y amable-grosero)

4- Escala habla como yo- habla diferente

5- Escala habla correctamente-habla incorrectamente

Siguiendo el proceso de la elaboración del instrumento, se sorteó el orden en que aparecerían las escalas, así como la colocación (a la derecha o a la izquierda) del polo positivo y del polo negativo en cada escala. De esta manera, el ordenamiento resultó totalmente aleatorio.

El formulario que recibió cada juez consta de 8 hojas que presentan el instrumento y una breve instrucción de uso (una para cada hablante del estímulo), y de una hoja de datos personales del juez al final.

\subsection{Confección del estímulo}

Tomando en cuenta las variables: REGION, SEXO y EDAD, se escogieron 8 hablantes según las siguientes características:

\section{Clase Media Alta Clase media baja}

Variedad A Colegio Calasanz Liceo Vargas Calvo hombres 6 jueces 5 jueces

mujeres 4 jueces 5 jueces

Variedad B Colegio Santa Ana Liceo Laboratorio hombres 5 jueces 5 jueces

mujeres 5 jueces 5 jueces

En el análisis de los datos, los colegios serán identificados según las siguientes siglas: 
Colegio Calasanz CC Liceo Vargas Calvo VC Colegio Santa Ana SA Liceo Laboratorio LL

\section{Descripción de los datos}

\subsection{Evaluación de los hablantes individualmente}

En este primer grupo de datos, se compararon las diversas evaluaciones que obtuvo cada hablante por colegios. Dicha comparación se llevó a cabo en los siguientes rubros:

1-Escala urbano-campesino (Gráfico 1-1)

2-Eje estatus social (Gráfico 1-2)

3-Eje emotivo (Gráfico 1-3)

4-Escala habla como yo-habla diferente (Gráfico 1-4)

5-Escala habla correctamente-habla incorrectamente (Gráfico 1-5)

A partir de los gráficos, se puede observar que no existen diferencias significativas para los hablantes 2, 3, 4, 6 y 7, mientras que para los hablantes 1,5 y 8 , las diferencias se muestran al tamente significativas.

En el caso de la hablante 1 (mujer menor, variedad A), se comprobó que los valores otorgados por todos los jueces fueron los más positivos y los más homogéneos de la totalidad de hablantes.

La hablante 5 (mujer menor, variedad B) recibió los valores más negativos en todos los rubros, excepto en el eje de emotividad. Creemos que una explicación posible para este hecho es la condescendencia emotiva de parte de los jueces debida a la historia que la hablante narró.

El hablante 8 (hombre mayor, variedad B) recibió los valores más heterogéneos o divergentes para todos los rubros, excepto para el eje emotivo.

De este primer grupo de datos, concluimos lo siguiente:

1- La hablante 1 recibió las calificaciones más positivas y más homogéneas debido a que:

a- es del Valle Central,

b- es una hablante de alto estatus $y$ alto nivel educativo, $y$

c- es una hablante del grupo de los jóvenes.
Por estas razones, la hablante 1 fue la que despertó una mayor identificación de grupo en todos los jueces, como se puede apreciar en el gráfico 1-4 (escala habla como yo-habla diferente).

2- La hablante 5 recibió las calificaciones más negativas y más homogéneas debido a que:

a- es de Guanacaste, y

b- es la hablante de menor estatus y menor nivel educativo.

La calificación positiva en el eje emotivo, en comparación con los rubros restantes, puede interpretarse, reiteramos, como la identificación de los jueces no tanto hacia el habla en sí como hacia la historia desarrollada por los hablantes.

3- El hablante 8 recibió valores más positivos de los colegios del Valle Central que los de Guanacaste debido a que:

a- es el hablante de mayor nivel educativo, por lo que los colegios del Valle Central calificaron más positivamente, $y$

b- es de Guanacaste, por lo que los colegios de esa región calificaron más negativamente.

La calificación en el eje emotivo para este hablante fue homogénea, resultando en un valor medio.

\subsection{Evaluación de los hablantes según la variante regional que representan (1)}

Los datos comprueban que todos los colegios calificaron con valores más positivos a los hablantes del Valle Central que a los de la región de Guanacaste en todos los rubros. Sin embargo, en el caso de LL, que representa la clase media baja de la región de Guanacaste, la diferencia de calificación resultó significativa en todos los ejes. (Se hace una salvedad para la escala habla correctamentehabla incorrectamente, en la cual el VC calificó más positivamente a loa hablantes de la región de Guanacaste. Sin embargo, por tratarse de una diferencia no significativa, no se considerará pertinente).

El hecho más relevante que a partir de la presente investigación se ha definido, queda comprobado en este segundo grupo de datos. A partir de los mismos, llegamos a la siguiente conclusión: el LL, que representa a la clase media baja de la región de Guanacaste, muestra una gran inseguridad 
lingü ística, lo cual se comprueba con la calificación significativamente más positiva para los hablantes de la variedad A. Por otra parte, los hablantes de la variedad B fueron calificados con valores más negativos.

Otros datos reveladores de la inseguridad lingü ística de los jueces de Guanacaste se dan en la calificación de los hablantes 1,5 y 8 (v. 5.1.), con los cuales se muestra la particularidad de un fenómeno que se evidencia como general.

Estas pruebas pueden ser corroboradas en los siguientes gráficos; en ellos la línea discontinua, que representa a los hablantes de la variedad $\mathrm{A}$, en todos los casos se encuentra por encima de la línea continua, que representa a los hablantes de la variedad $\mathrm{B}$.

(1) La siguiente "tabla de significación" muestra los valores de $\mathrm{T}$ cal. obtenidos en cada uno de los rubros. Los espacios en blanco corresponden a valores que no resultaron significativos.

$\begin{array}{lcccc} & \text { CC } & \text { VC } & \text { SA } & \text { LL } \\ \text { Escala urbano-campesino } & 1.92 & 2.05 & 0.51 & 5.33 \\ \text { Eje de estatus } & - & - & - & 4.08 \\ \text { Eje emotivo } & 1.55 & 3.74 & 2.62 & 5.60 \\ \begin{array}{l}\text { Escala habla como } \\ \text { yo-habla diferente }\end{array} & - & - & - & 2.34 \\ \begin{array}{l}\text { Escala habla correctamente } \\ \text { habla incorrectamente }\end{array} & - & - & - & 2.70\end{array}$

\section{Conclusiones}

Mediante la presente investigación, hemos comprobado que en la muestra estudiada correspondiente a la clase media baja de la región de Guanacaste existe una gran inseguridad lingü ística, producto de un factor de doble carácter: social-geográfico. Al pertenecer a una clase social en ascenso, estos jueces evalúan más positivamente la variedad de prestigio o estándar (la del Valle Central), mientras que la propia, que es una variedad regional, recibe valores más negativos.

Este debilitamiento en la identidad lingüística puede ser efecto de la creciente comunicación entre ambas regiones, lo cual conduce a que los hablantes de la variedad B adquieran conocimientos lingüísticos cada vez mayores sobre la variedad $\mathrm{A}$, reconocida como el habla de la "urbe".

La región de Guanacaste ha venido experimentando un fuerte desarrollo agro-industrial en los últimos años, provocando que sus habitantes aspiren a establecer en su región un nivel de vida urbano. Por ello, no es de extrañar la tendencia de una clase en ascenso por preferir los patrones urbanos, empezando por los lingüísticos. 
Gráfico 1-1

Escala urbano - campesino
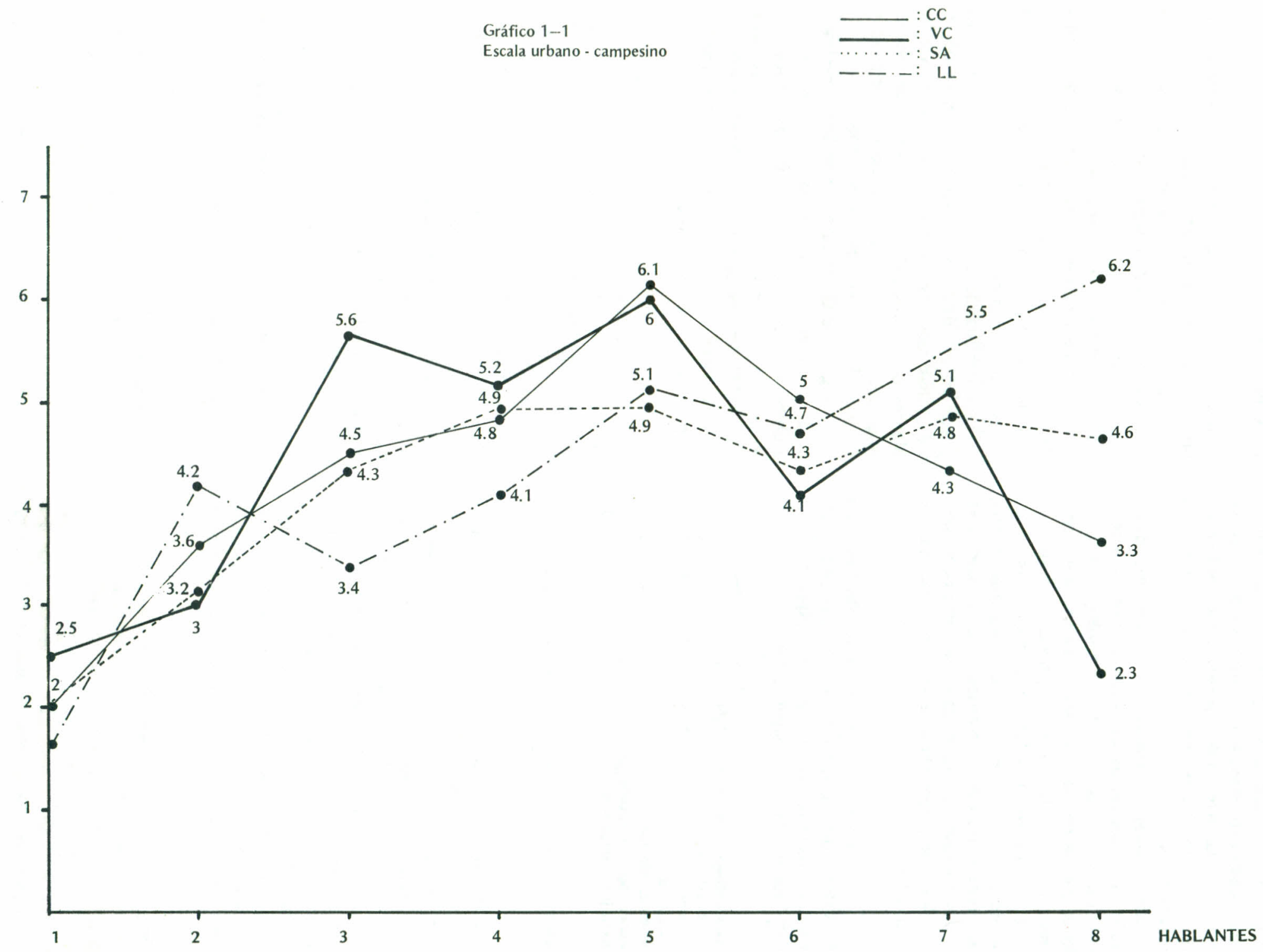

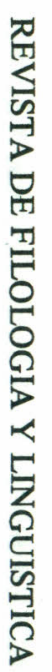


Gráfico 1-2

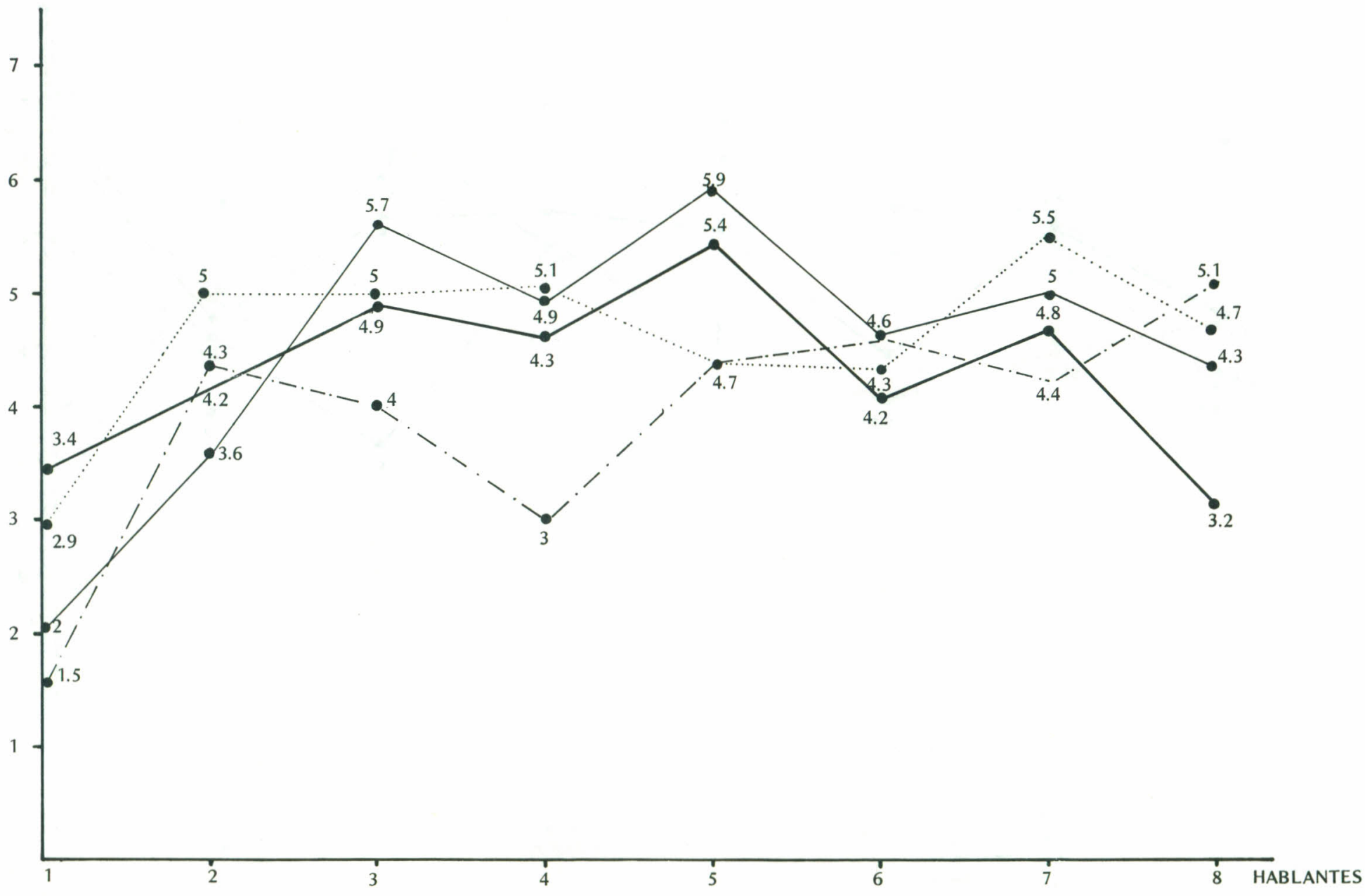


Gráfico 1-3

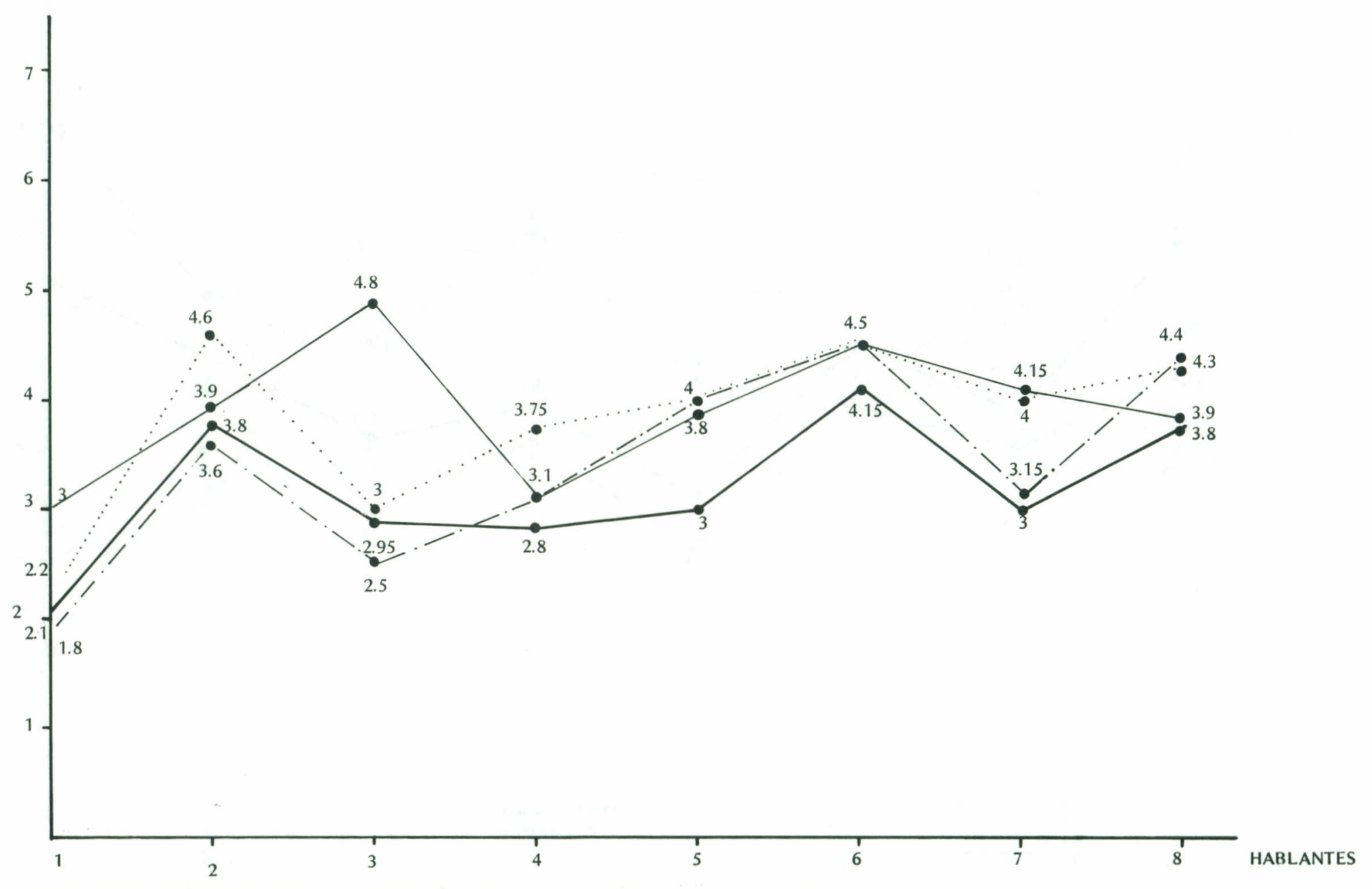


Gráfico 1-4

Escala habla como yo -

habla diferente

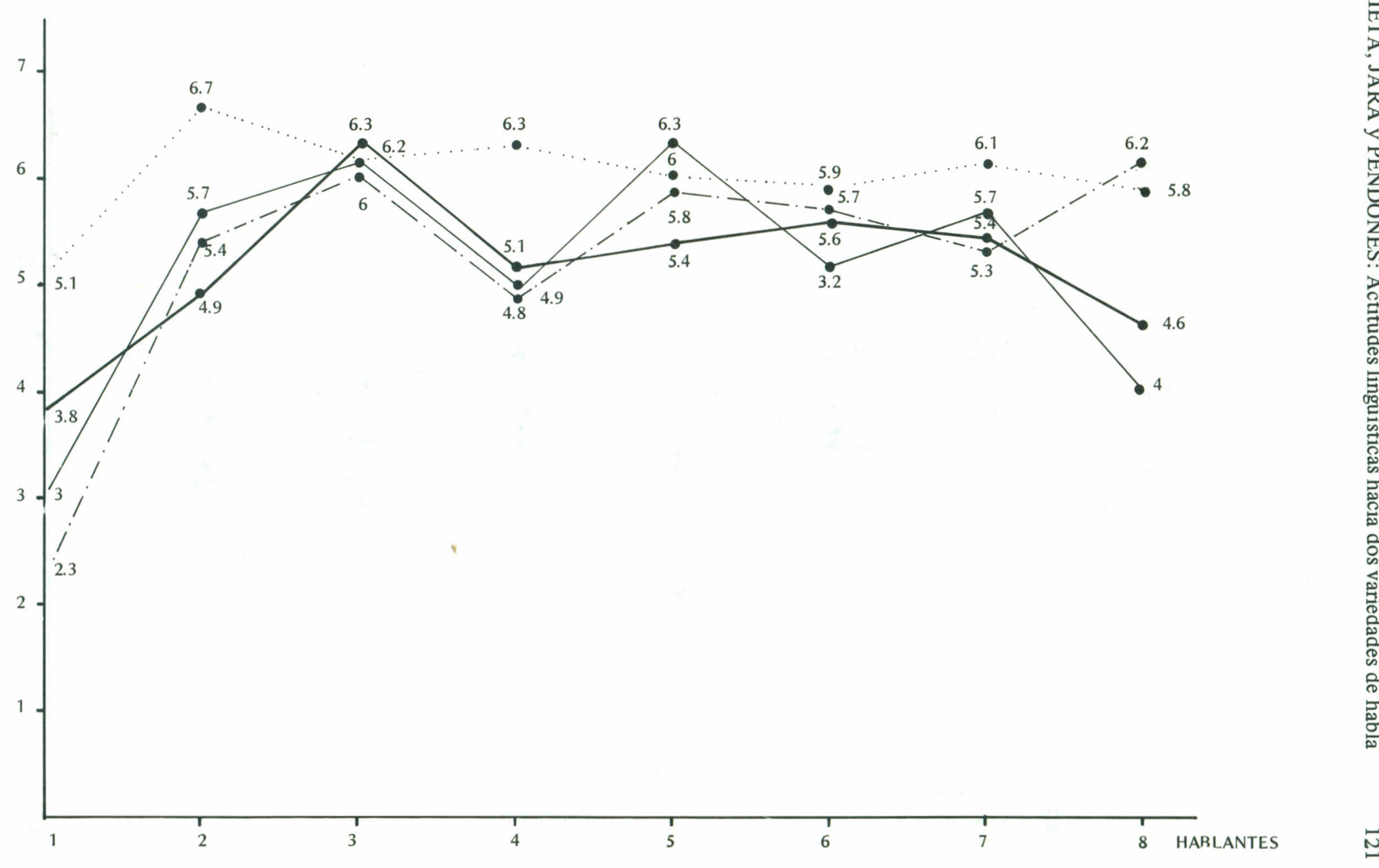


Gráfico 1-5

Escala habla correctamente

habla incorrectamente

$$
\begin{aligned}
& \longrightarrow: \mathrm{CC} \\
& \cdots \cdots \text { : SA }
\end{aligned}
$$

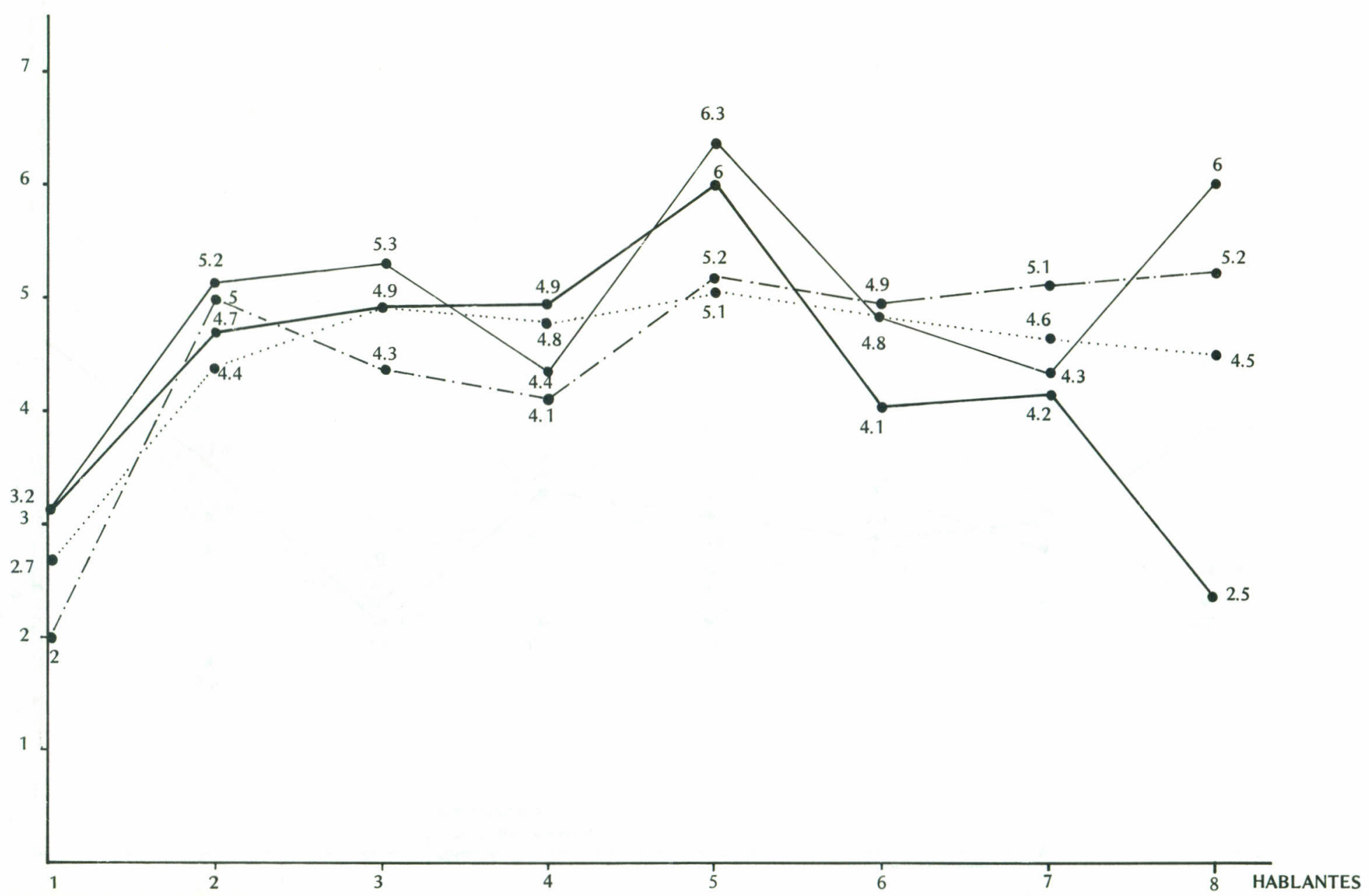

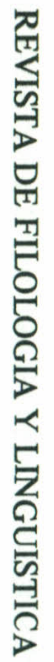




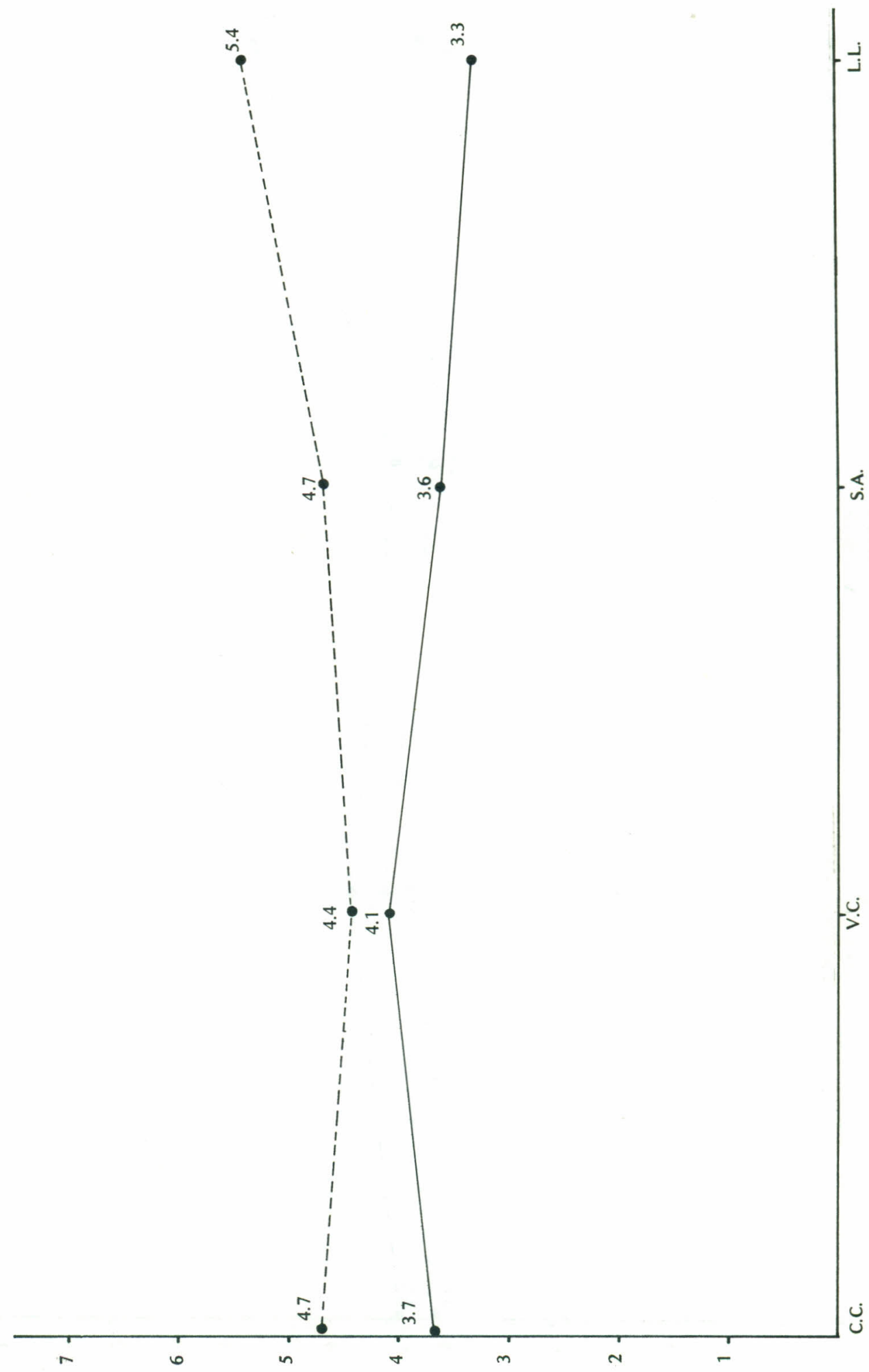


Gráfico 2-2

Eje estatus social

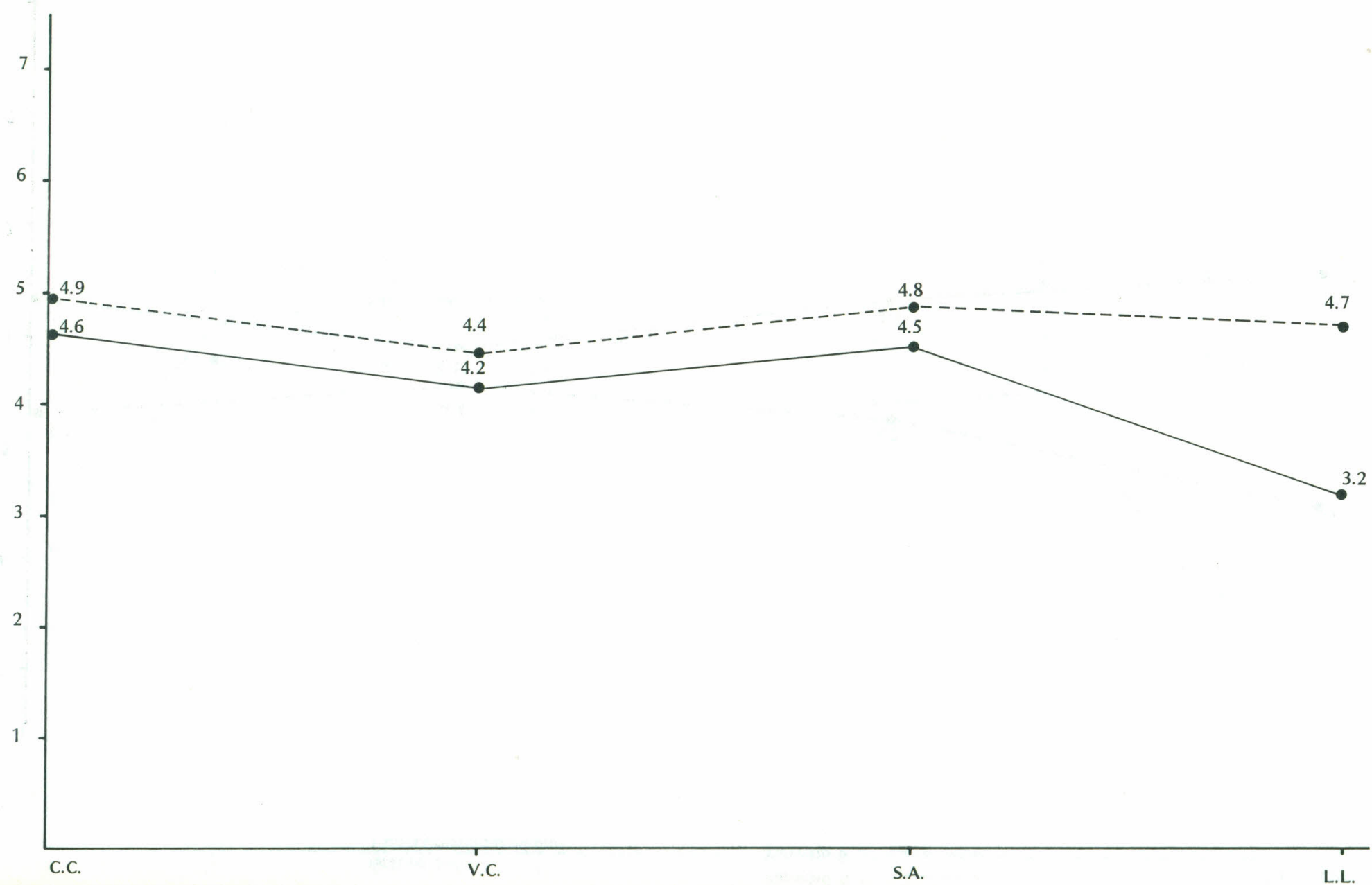




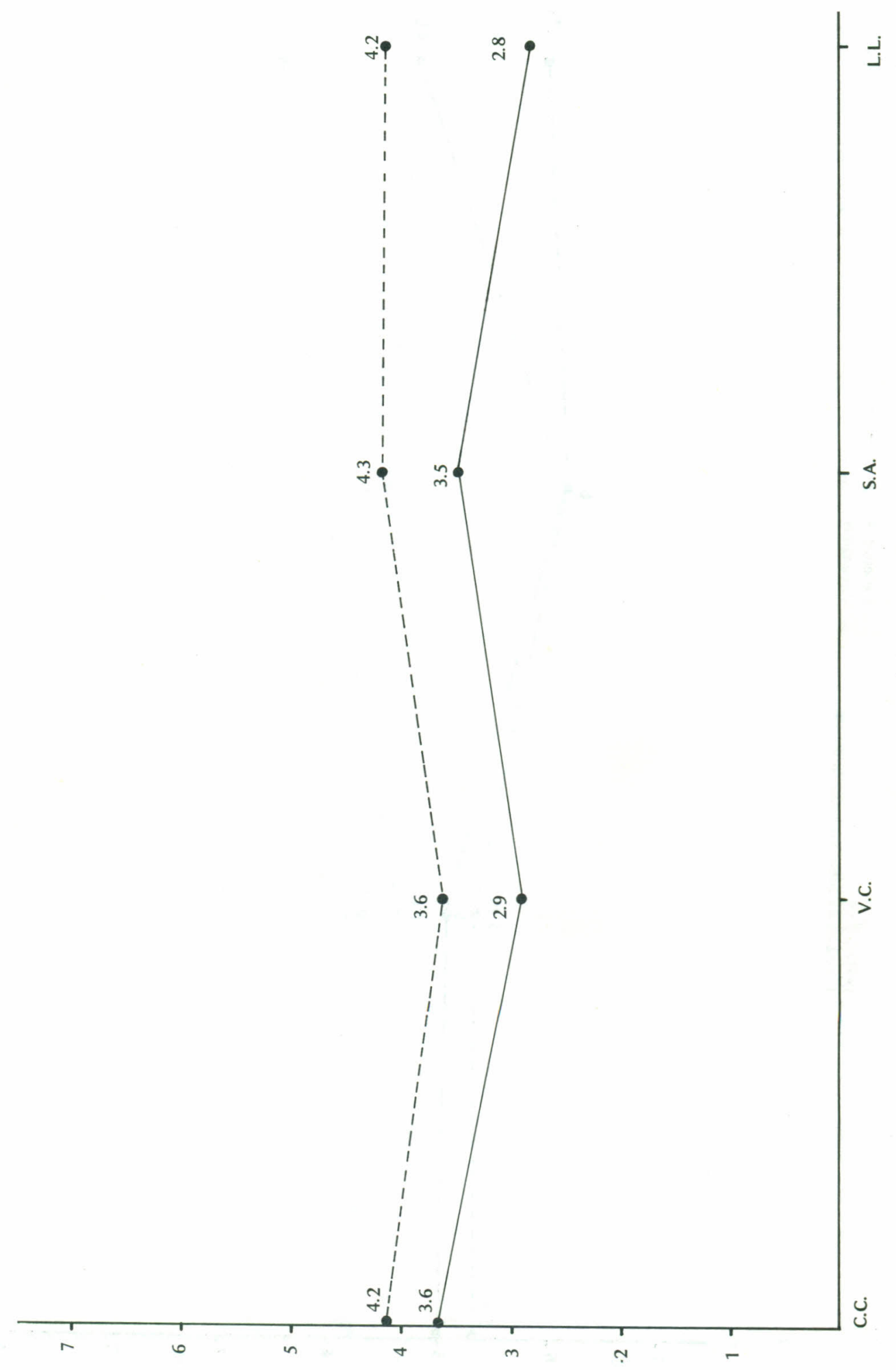




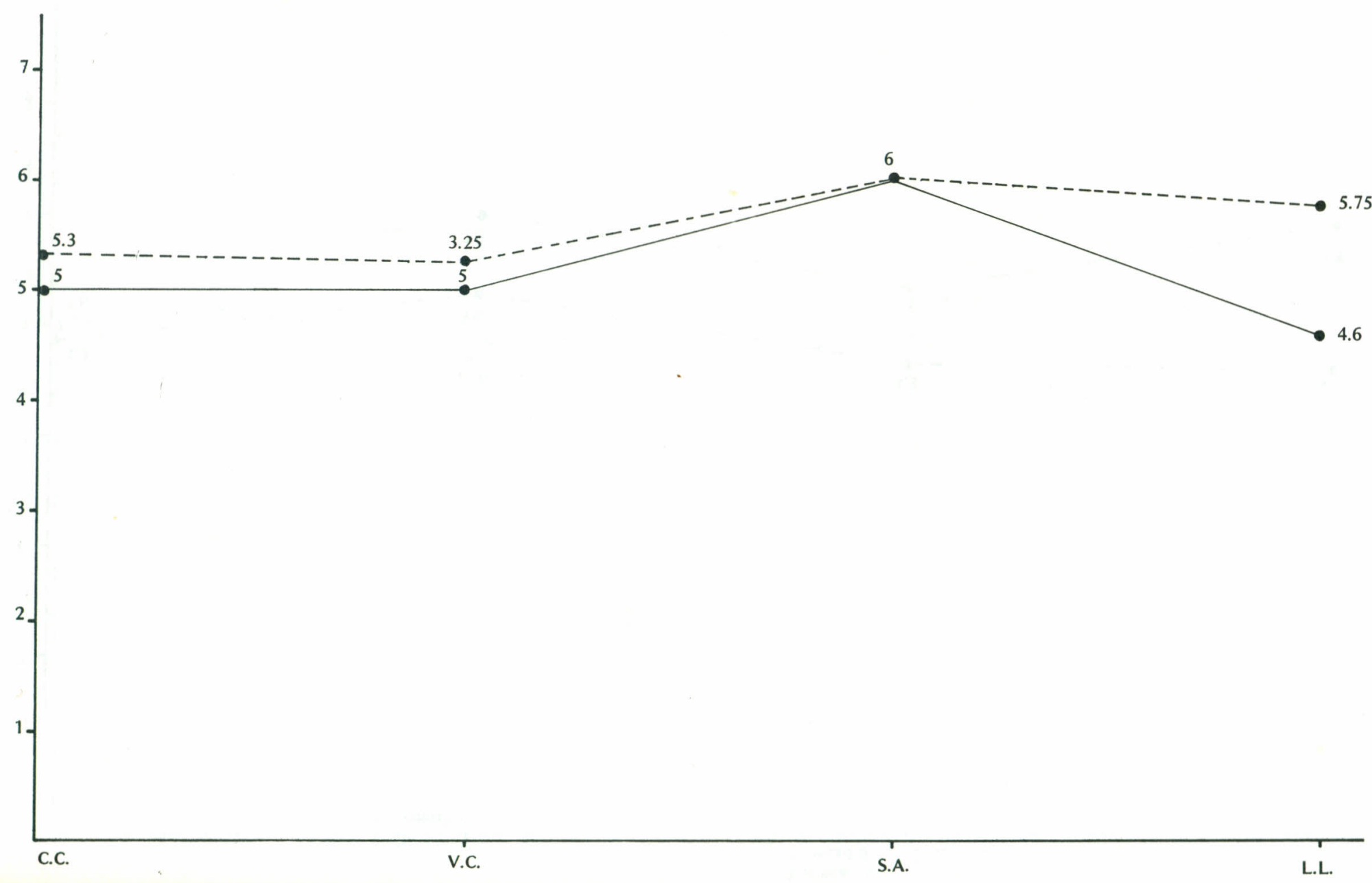


Gráfico 2-5

Escala habla correctamente

habla incorrectamente

Variedad B

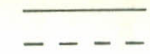

5.35

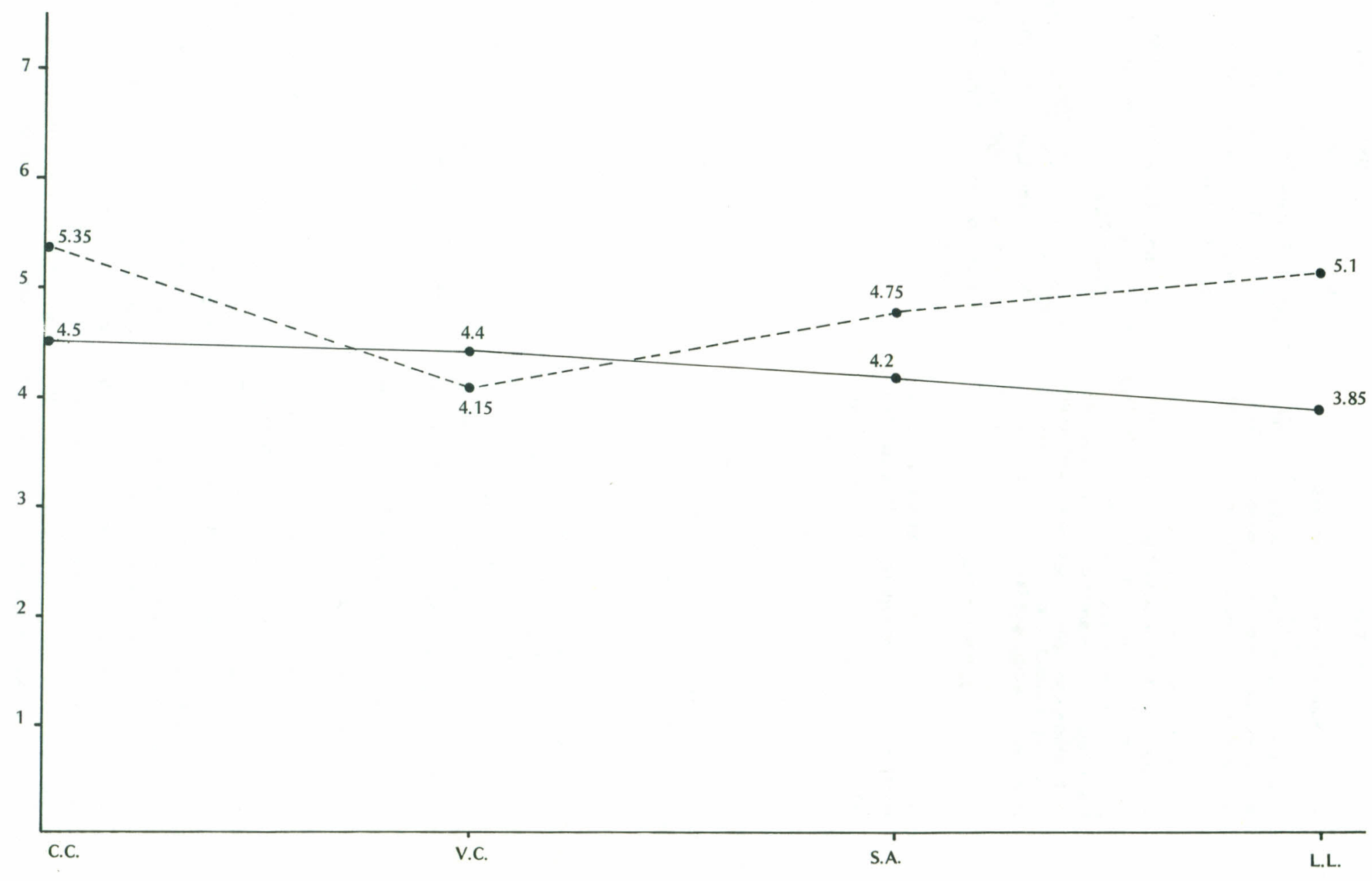




\section{NOTAS}

(1) Dr. Víctor Sánchez. Comunicación personal.

(2) En el análisis cuantitativo de los datos se utilizó como punto de referencia para establecer diferencias significativas la prueba $\mathrm{T}$ dif.: $\overline{\mathrm{x}}_{1}-\overline{\mathrm{x}}_{2}$, cuyo $\sigma$

resultado fue comparado con los valores establecidos para un grado de libertad 8 (grupos de 10 sujetos): $\mathrm{T}$ tab. 3.355 , con un margen de error de .01 , y 2.306 , con un margen de error de .05 .

Finalmente, hacemos notar que hablamos de valores positivos $y$ de valores negativos tomando el polo positivo como 1 y el polo negativo como 7 , según los siete espacios de la escala de Osgood.

\section{BIBLIOGRAFIA}

1- Bolaño, Sara. 1982. Introducción a la teoría y práctica de la sociolingüística. México: Trillas.
2- Hudson, R.A. 1981. La sociolingüistica. Barcelona: Anagrama.

3- Labov, William. 1972a. Sociolonguistic Patterns. University of Pennsylvania Press. (Versión española: Modelos sociolingüísticos. Madrid: Cátedra. 1983).

4- Meléndes, Carlos. 1978. Costa Rica: tierra y poblamiento en la Colonia. San José: Editorial Costa Rica.

5- Wölck, Wolfgang. 1973. "Attitudes toward Spanish and Quechua". En: Shuy \& Fasold. 1973. Language attitudes: current trends and prospects. Washington DC: Georgetown University Press. 